\title{
The Cauchy problem for hyperbolic systems with Hölder continuous coefficients with respect to the time variable
}

\author{
KUniHIKo KaJitani AND YASUO YuZAWA
}

\begin{abstract}
We discuss the local existence and uniqueness of solutions of certain nonstrictly hyperbolic systems, with Hölder continuous coefficients with respect to time variable. We reduce the nonstrictly hyperbolic systems to the parabolic ones and by use of the Tanabe-Sobolevski's method and the Banach scale method we construct a semi-group which gives a representation of the solution to the Cauchy problem.
\end{abstract}

Mathematics Subject Classification (2000): 35L45 (primary); 35A08 (secondary).

\section{Introduction}

We consider the following Cauchy problem:

$$
\left\{\begin{aligned}
\partial_{t} u(t, x)= & \sum_{j=1}^{d} A_{j}(t, x) \partial_{j} u(t, x) \\
& +B(t, x) u(t, x)+f(t, x), \quad \text { in }[0, T] \times \mathbb{R}^{d} \\
u(0, x)= & u_{0}(x), \quad x \in \mathbb{R}^{d},
\end{aligned}\right.
$$

where each $A_{j}$ and $B$ are $N \times N$ matrix functions, $f, u$ and $u_{0}$ are $N$ component vector functions and $\partial_{t}=\partial / \partial t, \partial_{j}=\partial / \partial x_{j}$. We assume that this system has weak hyperbolicity, that is,

(A.I) All eigenvalues of $\sum_{j=1}^{d} A_{j}(t, x) \xi_{j}$ are real valued in $[0, T] \times \mathbb{R}_{x}^{d} \times\left\{\mathbb{R}_{\xi}^{d} \backslash\{0\}\right\}$ and their multiplicity does not exceed $v$.

Many papers are devoted to the study of wellposedness in the Gevrey classes for the Cauchy problem (1.1). When all $A_{j}$ are smooth enough with respect to $t$, then this property was proved for the order $1 \leq s<1+1 /(v-1)$ by M. D. Bronstein in [1] in

Received July 10, 2006; accepted in revised form October 16, 2006. 
the higher order scalar case and by K. Kajitani in [4] in the system case, respectively. Moreover they have shown it in the case that the coefficient also depend on $x$. When each $A_{j}$ has only $\mu$-Hölder continuity in $t$ for some $0<\mu \leq 1$, the Cauchy problem is also wellposed in the Gevrey classes but the Gevrey order must be lower than the smooth case. The first result in the Hölder continuous case was derived by F. Colombini, E. Jannelli and S. Spagnolo in [2]. They proved that the Cauchy problem to the second order equation $u_{t t}=a(t) u_{x x}$ was Gevrey wellposed for the order $1 \leq s<1+\mu / 2$ and, it is important, this order is optimal. T. Nishitani in [8] extended to the second order equations with coefficients also depending on $x$, and then Y. Ohya and S. Tarama in [9] extended that the higher order scalar equation was Gevrey wellposed for $1 \leq s<1+\mu / \nu$. The system case was investigated by Kajitani in [5], and he showed that the weakly hyperbolic systems were wellposed in the Gevrey classes for $1 \leq s<1+\mu /(v+1)$.

When the coefficients depend only on $t$, D'Ancona, T. Kinoshita and Spagnolo in [3] proved the Gevrey wellposedness for $1 \leq s<1+\mu / v$ to $3 \times 3$ weakly hyperbolic systems with coefficients depending on $t$. To prove it, they derived the energy estimates for the approximate symbols and moreover Yuzawa in [11] has treated the general systems of which coefficients depend only on time variable.

In this paper, we shall extend their result to any $N \times N$ system whose coefficients depend also on the space variables by using the other approach, semi-group method called Tanabe-Sobolevski method (cf. $[6,10]$ ) and consequently obtain the energy estimates.

To state our results we shall introduce the Gevrey classes and their properties.

Definition 1.1. Let $s \geq 1$, then we denote by $\gamma^{(s)}\left(\mathbb{R}^{d}\right)$ the set of all functions satisfying the following condition: for any compact subset $K$ of $\mathbb{R}^{d}$, there exist constants $C_{K}>0$ and $A_{K}>0$ such that

$$
\left|\partial_{x}^{\alpha} u(x)\right| \leq C_{K} A_{K}^{|\alpha|}|\alpha| !^{s}
$$

for any $x \in K$ and $\alpha \in \mathbb{N}^{d}$ and we define $\gamma_{0}^{(s)}\left(\mathbb{R}^{d}\right)=\gamma^{(s)}\left(\mathbb{R}^{d}\right) \cap C_{0}\left(\mathbb{R}^{d}\right)$.

Definition 1.2. Let $k$ be an integer and $0<\mu \leq 1$. For a Banach space $Y$, we denote by $C^{k, \mu}([0, T] ; Y)$ the set of functions $u(t)$ which are $k$ times differentiable in $Y$ with respect to $t$ and $(\partial / \partial t)^{k} u(t)$ are $\mu$-Hölder continuous in $Y$ : there exists a constant $C>0$ such that

$$
\left\|\partial_{t}^{l} u(t)\right\|_{Y} \leq C \quad(0 \leq l \leq k), \quad\left\|\partial_{t}^{k} u(t)-\partial_{t}^{k} u\left(t^{\prime}\right)\right\|_{Y} \leq C\left|t-t^{\prime}\right|^{\mu}
$$

for $t, t^{\prime} \in[0, T]$. We write $C^{k, 0}([0, T] ; Y)$ as $C^{k}([0, T] ; Y)$ in brief.

Definition 1.3. For $\rho \geq 0, s>1, h>0$ and $l \in \mathbb{R}$, we define

$$
H_{\Lambda(\rho)}^{l}\left(\mathbb{R}^{d}\right)=\left\{u \in L_{x}^{2}\left(\mathbb{R}^{d}\right) ;\langle\xi\rangle_{h}^{l} e^{\Lambda(\rho)} \hat{u}(\xi) \in L_{\xi}^{2}\left(\mathbb{R}^{d}\right)\right\},
$$


where $\langle\xi\rangle_{h}=\sqrt{h^{2}+|\xi|^{2}}, \Lambda(\rho)=\Lambda(\rho, \xi ; s, h)=\rho\langle\xi\rangle_{h}^{1 / s}$ and $\hat{u}(\xi)$ stands for a Fourier transform of $u(x)$ :

$$
\hat{u}(\xi)=\int_{\mathbb{R}^{d}} e^{-i x \cdot \xi} u(x) d x,
$$

and for $\rho<0$ we define $H_{\Lambda(\rho)}^{l}\left(\mathbb{R}^{d}\right)$ as the dual space of $H_{\Lambda(-\rho)}^{-l}\left(\mathbb{R}^{d}\right)$.

When $\rho=0, H_{\Lambda(0)}^{l}=H_{0}^{l}$ is a usual Sobolev space and we write them as $H^{l}$ in brief. $H_{\Lambda(\rho)}^{l}$ is a Hilbert space with inner product

$$
(u, v)_{H_{\Lambda(\rho)}^{l}}=\left(\langle\xi\rangle^{l} e^{\rho\langle\xi\rangle_{h}^{1 / s}} u,\langle\xi\rangle^{l} e^{\left.\rho\langle\xi\rangle_{h}^{1 / s} v\right)_{L^{2}}}\right.
$$

and we define the norm of $H_{\Lambda(\rho)}^{l}$ by $\|u\|_{H_{\Lambda(\rho)}^{l}}=\left\|\langle\xi\rangle^{l} e^{\rho\langle\xi\rangle_{h}^{1 / s}} \hat{u}(\xi)\right\|_{L^{2}}$.

Definition 1.4. We define $e^{\Lambda(\rho)}=e^{\rho\langle D\rangle_{h}^{1 / s}}$ a pseudo differential operator of order infinity such as

$$
e^{\Lambda(\rho)} u(x)=e^{\rho\left\langle D_{x}\right\rangle_{h}^{1 / s}} u(x)=\frac{1}{(2 \pi)^{d}} \int_{\mathbb{R}^{d}} e^{i x \cdot \xi+\Lambda(\rho, \xi)} \hat{u}(\xi) d \xi
$$

for $u(x)$ in $H_{\Lambda(\rho)}^{l}$.

Definition 1.5. Let $\rho(t)$ be a positive definite function in $[0, T], k$ an integer $\geq$ $0,0<\mu \leq 1$ and $l \in \mathbb{R}$. Then, we denote by $e^{-\Lambda(\rho(t))} C^{k, \mu}\left([0, T] ; H^{l}\right)$ the class of functions $f(t, x)$ for which to every $t \in[0, T]$,

$$
e^{\Lambda(\rho(t))} f(t, x)=e^{\rho(t)\left\langle D_{x}\right\rangle^{1 / s}} f(t, x) \in C^{k, \mu}\left([0, T] ; H^{l}\right) .
$$

We note the relations between $\gamma_{0}^{(s)}\left(\mathbb{R}^{d}\right)$ and $H_{\Lambda(\rho)}^{l}\left(\mathbb{R}^{d}\right)$.

Proposition 1.6 (cf. Lemma 1.2 in [4]). For any $u(x)$ in $\gamma_{0}^{(s)}\left(\mathbb{R}^{d}\right)$ and $l \in \mathbb{R}$, there exists a constant $\rho_{u}>0$ such that $u(x)$ in $H_{\Lambda\left(\rho_{u}\right)}^{l}\left(\mathbb{R}^{d}\right)$.

Conversely, if $u(x)$ belongs to $H_{\Lambda(\rho)}^{l}\left(\mathbb{R}^{d}\right)$ for some $\rho>0$, then $u$ belongs to $\gamma^{(s)}\left(\mathbb{R}^{d}\right)$.

Now, we shall state the main theorems.

Theorem 1.7. Let $1 \leq s<1+\mu / \nu, \sigma=(\nu-1)(1-1 / s)$ and $0<\mu \leq 1$ and take $\delta \geq 0$ such that $s(\delta+\sigma+1)>1+\mu$. Assume that (A.I) and the following condition (A.II) are valid;

(A.II) each $A_{j}(t, x)$ belongs to $C^{0, \mu}\left([0, T] ; \gamma^{(s)}\left(\mathbb{R}^{d}\right)\right)$ for $j=1, \ldots, d$ and $B(t, x) \in C^{0}\left([0, T] ; \gamma^{(s)}\left(\mathbb{R}^{d}\right)\right)$. 
Then for every $u_{0}(x)$ in $H_{\Lambda\left(T+\rho_{0}\right)}^{l}\left(\mathbb{R}^{d}\right)$ and for every $f(t, x)$ in $e^{-\Lambda\left(T-t+\rho_{0}\right)} C([0, T]$; $\left.H^{l}\left(\mathbb{R}^{d}\right)\right)$, there exists a unique solution $u(t, x)$ of the Cauchy problem $(1.1)$, which is in $e^{-\Lambda(T-t)} C\left([0, T] ; H^{l+\delta}\left(\mathbb{R}^{d}\right)\right) \cap e^{-\Lambda(T-t)} C^{1}\left([0, T] ; H^{l-1+\delta}\left(\mathbb{R}^{d}\right)\right)$ and satisfies

$$
\|u(t, \cdot)\|_{H_{\Lambda(T-t)}^{l+\delta}} \leq C\left(\left\|u_{0}\right\|_{H_{\Lambda\left(T+\rho_{0}\right)}^{l}}+\int_{0}^{t}\|f(r, \cdot)\|_{H_{\Lambda\left(T-r+\rho_{0}\right)}^{l}} d r\right),
$$

for any $l \in \mathbb{R}$ and $0 \leq t \leq T$.

Considering the property of the finite propagation of the solution for the weakly hyperbolic system and Proposition 1.6, the following theorem is concluded by Theorem 1.7.

Theorem 1.8. Assume that (A.I) and (A.II). If $1 \leq s<1+\mu / v$ and $0<\mu \leq 1$, then for any $f(t, x)$ in $C\left([0, T] ; \gamma^{(s)}\left(\mathbb{R}^{d}\right)\right)$ and $u_{0}(x)$ in $\gamma^{(s)}\left(\mathbb{R}^{d}\right)$, there is a unique solution $u(t, x)$ in $C^{1}\left([0, T] ; \gamma^{(s)}\left(\mathbb{R}^{d}\right)\right)$ of the equation $(1.1)$.

\section{Preliminaries}

In this section we shall introduce some notation and fundamental propositions on the pseudo differential operator theory.

We denote by $S_{\rho, \delta, l}^{m}(0 \leq \delta<\rho \leq 1)$ a class of symbols $p(x, \xi)$ satisfying

$$
|p|_{S_{\rho, \delta, l}^{m}}=\sup _{(x, \xi) \in \mathbb{R}^{2 d},|\alpha+\beta| \leq l} \frac{\left|p_{(\beta)}^{(\alpha)}(x, \xi)\right|}{\langle\xi\rangle^{m-\rho|\alpha|+\delta|\beta|}}<\infty,
$$

where $p_{(\beta)}^{(\alpha)}(x, \xi)=D_{x}^{\beta} \partial_{\xi}^{\alpha} p(x, \xi)$. We write $S_{1,0, l}^{m}$ as $S_{l}^{m}$ in brief.

We denote by $\left(S_{\rho, \delta, l}^{m}\right)^{N \times N}$ a class of matrix symbols $P(x, \xi)=\left(p_{i j}(x, \xi)\right)_{1 \leq i, j \leq N}$ such that all $p_{i j}$ are in $S_{\rho, \delta, l}^{m}$ and we define

$$
|P|_{S_{\rho, \delta, l}^{m}}=\max _{1 \leq i, j \leq N}\left|p_{i j}\right|_{S_{\rho, \delta, l}^{m}}
$$

We often write $\left(S_{\rho, \delta, l}^{m}\right)^{N \times N}$ as $S_{\rho, \delta, l}^{m}$ in brief and $S_{\rho, \delta}^{m}=\cap_{l} S_{\rho, \delta, l}^{m}$.

For $p(x, \xi) \in S_{\rho, \delta}^{m}$, we define the pseudo differential operator $p\left(x, D_{x}\right)$ :

$$
p\left(x, D_{x}\right) u(x)=\frac{1}{(2 \pi)^{d}} \int e^{i x \xi} p(x, \xi) \hat{u}(\xi) d \xi
$$

The following proposition is well known as the boudedness in $H^{q}$ of pseudo differential operators. 
Proposition 2.1. Let $0 \leq \delta<\rho \leq 1$ and

$$
M_{0}(q)=\left[\frac{2\left(|q|+l_{0}+d+1\right)}{2-\delta}\right]+l_{0}, \quad l_{0}=\left[\frac{\delta(d+1)}{\rho-\delta}\right]+1 .
$$

Assume that $l \geq M_{0}(q)$ and $p(x, \xi)$ be in $S_{\rho, \delta, l}^{m}$. Then, we have

$$
\left\|p\left(x, D_{x}\right) u\right\|_{H^{q}} \leq C_{q}|p|_{S_{\rho, \delta, M_{0}(q)}^{m}}\|u\|_{H^{q+m}}
$$

for $u \in H^{q+m}$.

Next, we denote by $\Gamma_{s, r, l}^{m}$ a class of symbols $p(x, \xi)$ satisfying

$$
|p|_{\Gamma_{s, r, l}^{m}}=\sup _{(x, \xi) \in \mathbb{R}^{2 d},|\alpha+\beta| \leq l, \gamma \in \mathbb{Z}_{+}^{d}} \frac{\left|p_{(\beta+\gamma)}^{(\alpha)}(x, \xi)\right|}{r^{|\gamma|}|\gamma| !^{s}\langle\xi\rangle^{m-|\alpha|}}<\infty .
$$

For a symbol $p(x, \xi) \in \Gamma_{s, r, l}^{m}$, we define an operator $p_{\Lambda(\rho)}\left(x, D_{x}\right)$ :

$$
p_{\Lambda(\rho)}\left(x, D_{x}\right)=e^{\Lambda(\rho)} p\left(x, D_{x}\right) e^{-\Lambda(\rho)},
$$

where $\Lambda(\rho)=\rho\left\langle D_{x}\right\rangle_{h}^{1 / s}$. It follows from Kumano-go's formula that the symbol of $p_{\Lambda(\rho)}\left(x, D_{x}\right)$ give by

$$
\sigma\left(p_{\Lambda(\rho)}\right)(x, \xi)=\frac{1}{(2 \pi)^{d}} \text { Os- } \iint_{\mathbb{R}^{2 d}} e^{-i y \eta+\Lambda(\xi+\eta)-\Lambda(\xi)} p(x+y, \xi) d y d \eta,
$$

where Os- $\iint$ means an oscillatory integral. We denote by $p_{\Lambda}(x, \xi)$ the symbol of $p_{\Lambda(\rho)}\left(x, D_{x}\right)$ in brief.

From (2.3) and Taylor's formula,

$$
p_{\Lambda}(x, \xi)=p(x, \xi)+\sum_{0<|\gamma|<N} p_{(\gamma)}(x, \xi) \omega_{\gamma}(\rho, \xi)+r_{N}(p)(x, \xi)
$$

where

$$
\omega_{\gamma}(\rho, \xi)=\frac{1}{\gamma !} e^{-\rho\langle\xi\rangle_{h}^{\kappa}} \partial_{\xi}^{\gamma}\left(e^{\rho\langle\xi\rangle_{h}^{\kappa}}\right)
$$

and

$$
\begin{aligned}
& r_{N}(p)(x, \xi)=\frac{N}{(2 \pi)^{d}} \\
& \times \sum_{|\gamma|=N} \operatorname{Os}-\iint_{\mathbb{R}^{2 d}} \int_{0}^{1}(1-\theta)^{N-1} e^{-i y \eta+\Lambda(\xi+\eta)-\Lambda(\xi)} p_{(\gamma)}(x+\theta y, \xi) \omega_{\gamma}(\rho, \xi+\eta) d \theta d y d \eta .
\end{aligned}
$$


Proposition 2.2 ([5]). Let $p(x, \xi)$ be in $\Gamma_{s, r, l}^{m}, \Lambda(\rho)=\rho\langle\xi\rangle_{h}^{1 / s}$ and $s>1$.

(i) $\omega_{\gamma}$ and $r_{N}(p)$ satisfy the following inequalities:

$$
\begin{aligned}
\left|\partial_{\xi}^{\alpha} \omega_{\gamma}(\rho, \xi)\right| & \leq C_{\alpha \gamma}\left(|\rho|+h^{-\kappa}\right)^{|\gamma|}\langle\xi\rangle_{h}^{-(1-\kappa)|\gamma|-|\alpha|}, \\
\left|\partial_{\xi}^{\alpha} D_{x}^{\beta} r_{N}(p)(x, \xi)\right| & \leq C_{\alpha \beta N}\left(|\rho|+h^{-\kappa}\right)^{N}\langle\xi\rangle_{h}^{m-N(1-\kappa)-|\alpha|},
\end{aligned}
$$

for $(x, \xi) \in \mathbb{R}^{2 d}, \alpha, \beta \in \mathbb{Z}_{+}^{d}$, where $\kappa=\frac{1}{s}$.

(ii) If $\rho$ satisfies

$$
|\rho| \leq\left(24^{s} d r\right)^{-1 / s}
$$

and l satisfies

$$
l \geq\left[\frac{s l^{\prime}}{s-1}\right]+l^{\prime}+\left[\frac{d}{2}\right]+1,
$$

then the symbol $p_{\Lambda}(x, \xi)$ belongs to $S_{l^{\prime}}^{m}$. Moreover, there exists a constant $C_{l^{\prime}}$ such that

$$
\left|p_{\Lambda}\right|_{l^{\prime}}^{m} \leq C_{l^{\prime}}|p|_{\Gamma_{s, r, l}^{m}}
$$

The following proposition is the fundamental property on the hyperbolic polynomial:

Proposition 2.3 ([1]). Let $p(t, x, \lambda, \xi)$ be a hyperbolic polynomial of order $N$, that is, $p$ can be factorized by real roots:

$$
p(t, x, \lambda, \xi)=\sum_{|\alpha|+j=N} a_{j, \alpha}(t, x) \lambda^{j} \xi^{\alpha}=\prod_{j=1}^{N}\left(\lambda-\lambda_{j}(t, x, \xi)\right),
$$

where all $\lambda_{j}(t, x, \xi)$ are real valued for $(t, x, \xi) \in[0, T] \times \mathbb{R}^{d} \times \mathbb{R}^{d}$. Assume that the multiplicity of $\lambda_{j}(t, x, \xi)$ is at most $v(\leq N)$ and all the coefficients $a_{j, \alpha}(t, x)$ and $D_{x}^{\beta} a_{j, \alpha}(t, x)(|\beta| \leq v)$ are bounded in $[0, T] \times \mathbb{R}^{d}$, then $p(t, x, \lambda, \xi)$ satisfies the following estimates:

$$
\begin{aligned}
\frac{1}{\left|p\left(t, x, \lambda-i\langle\xi\rangle_{h}^{\kappa}, \xi\right)\right|} \leq C\left(|\lambda|+\langle\xi\rangle_{h}\right)^{-N+v}\langle\xi\rangle_{h}^{-\kappa \nu}, \\
\frac{\left|p_{(\beta)}^{(\alpha)}\left(t, x, \lambda-i\langle\xi\rangle_{h}^{\kappa}, \xi\right)\right|}{\left|p\left(t, x, \lambda-i\langle\xi\rangle_{h}^{\kappa}, \xi\right)\right|} \leq C\langle\xi\rangle_{h}^{-\kappa|\alpha|+(1-\kappa)|\beta|} \quad(|\alpha+\beta| \leq v),
\end{aligned}
$$

for $\lambda \in \mathbb{C}$ such that $\operatorname{Im} \lambda<0$ and for $(x, \xi)$ in $[0, T] \times \mathbb{R}^{d}$. 


\section{The Cauchy problem in Sobolev spaces}

In this section we assume that $1 \leq s<1+\mu / \nu$ and put $\kappa=1 / s$.

We reintroduce the equation (1.1):

$$
\left\{\begin{array}{l}
P(t, x, D) u(t, x)=-i f(t, x), \quad(t, x) \in[0, T] \times \mathbb{R}^{d} \\
u(0, x)=u_{0}(x), \quad x \in \mathbb{R}^{d}
\end{array}\right.
$$

where

$$
P(t, x, D)=D_{t}-\sum_{j=1}^{d} A_{j}(t, x) D_{j}+i B(t, x),
$$

and $D=\left(D_{t}, D_{x}\right)=\left(D_{t}, D_{1}, D_{2}, \ldots, D_{d}\right), D_{t}=-i \partial_{t}$ and $D_{i}=-i \partial_{i}$.

For some non negative and continuously differentiable function $\rho(t)$, we set $v(t, x)=e^{\Lambda(\rho(t))} u(t, x)=e^{\rho(t)\left\langle D_{x}\right\rangle_{h}^{K}} u(t, x)$. Then we can reduce the problem (1.1) to

$$
\left\{\begin{array}{l}
P_{\Lambda}\left(t, x, D_{t}, D_{x}\right) v(t, x)=g(t, x) \\
v(0, x)=v_{0}(x)
\end{array}\right.
$$

where

$$
\begin{gathered}
D_{t, \Lambda}=D_{t, \Lambda(\rho(t))}=D_{t}+i \rho^{\prime}(t)\left\langle D_{x}\right\rangle_{h}^{\kappa}, \\
P_{\Lambda}\left(t, x, D_{t}, D_{x}\right)=D_{t, \Lambda} I-\sum_{j=1}^{d} A_{j, \Lambda}\left(t, x, D_{x}\right) D_{j}+i B_{\Lambda}\left(t, x, D_{x}\right),
\end{gathered}
$$

for $(t, x) \in \mathbb{R}^{1+d}, v_{0}(x)=e^{\Lambda(\rho(0))} u_{0}(x), g(t, x)=-i e^{\Lambda(\rho(t))} f(t, x)$, and each $A_{j, \Lambda}\left(t, x, D_{x}\right)$ and $B_{\Lambda}$ are pseudo differential operators such as

$$
\begin{gathered}
A_{j, \Lambda}\left(t, x, D_{x}\right)=A_{j, \Lambda(\rho(t))}\left(t, x, D_{x}\right)=e^{\rho(t)\left\langle D_{x}\right\rangle_{h}^{\kappa}} A_{j}(t, x) e^{-\rho(t)\left\langle D_{x}\right\rangle_{h}^{\kappa}}, \\
B_{\Lambda}\left(t, x, D_{x}\right)=B_{\Lambda(\rho(t))}\left(t, x, D_{x}\right)=e^{\rho(t)\left\langle D_{x}\right\rangle_{h}^{\kappa}} B(t, x) e^{-\rho(t)\left\langle D_{x}\right\rangle_{h}^{\kappa}}
\end{gathered}
$$

We shall solve the equation (3.1) in Sobolev space $H^{l}$ by using the semi group for $\left.\left.i \rho^{\prime}(t)\left\langle D_{x}\right\rangle_{h}^{\kappa}\right) I-A_{\Lambda}(t)\right)$.

\section{Construction of $\left.\left(\left(\lambda+i \rho^{\prime}(\tau)\left\langle D_{x}\right\rangle_{h}^{\kappa}\right)\right) I-A_{\Lambda}(\tau)\right)^{-1}$}

Let $\tau$ be fixed in $[0, T]$. In this section, we shall construct the inverse of $((\lambda+$ $\left.\left.i \rho^{\prime}(\tau)\left\langle D_{x}\right\rangle_{h}^{\kappa}\right) I-A_{\Lambda}(\tau)\right)$ for a complex number $\lambda$ with $\operatorname{Im} \lambda \leq-h_{0}$. We consider the following resolvent equation :

$$
\left(\left(\lambda+i \rho^{\prime}(\tau)\left\langle D_{x}\right\rangle_{h}^{\kappa}\right) I-A_{\Lambda}(\tau)\right) v=g,
$$


for $g \in H^{q}$, where

$$
A(\tau)=A\left(\tau, x, D_{x}\right)=\sum_{j=1}^{d} A_{j}(\tau, x) D_{j}
$$

and

$$
A_{\Lambda}(\tau)=A_{\Lambda(\rho(\tau))}\left(t, x, D_{x}\right)=\sum_{j=1}^{d} A_{j, \Lambda(\rho(\tau))}\left(\tau, x, D_{x}\right) D_{j} .
$$

Let us define several symbols as follows:

$$
\begin{aligned}
A(t, x, \xi) & =\sigma(A)(t, x, \xi)=\sum_{j=1}^{d} A_{j}(t, x) \xi_{j}, \\
A_{\Lambda}(t, x, \xi) & =\sigma\left(A_{\Lambda(\rho(t))}\right)(t, x, \xi)=\sum_{j=1}^{d} A_{j, \Lambda(\rho(t))}(t, x, \xi) \xi_{j}, \\
P(t, x, \lambda, \xi) & =\sigma(P)(t, x, \lambda, \xi)=\lambda I-\sum_{j=1}^{d} A_{j}(t, x, \xi) \xi_{j}+i B(t, x), \\
P_{\Lambda}(t, x, \lambda, \xi) & =\sigma\left(P_{\Lambda}\right)(t, x, \lambda, \xi) \\
& =\lambda_{\Lambda} I-\sum_{j=1}^{d} A_{j, \Lambda}(\rho(t))(t, x, \xi) \xi_{j}+i B_{\Lambda}(\rho(t))(t, x, \xi), \\
H(t, x, \lambda, \xi) & ={ }^{c o}\left(\lambda_{\Lambda} I-A_{\Lambda}(t, x, \xi)\right), \\
p(t, x, \lambda, \xi) & =\operatorname{det}(\lambda I-A(t, x, \xi)), \\
p_{L}(t, x, \lambda, \xi) & =\operatorname{det}\left(\lambda_{\Lambda} I-A_{\Lambda}(t, x, \xi)\right), \\
M(t, x, \lambda, \xi) & =\frac{1}{p\left(t, x, \lambda{ }_{\Lambda}, \xi\right)} I, \\
\lambda \Lambda & =\lambda+i \rho^{\prime}(t)\langle\xi\rangle_{h}^{\kappa} .
\end{aligned}
$$

We shall consider an operator $\tilde{P}\left(t, x, \lambda, D_{x}\right)=\left(\lambda I-A_{\Lambda}\left(t, x, D_{x}\right)\right) \circ H\left(t, x, \lambda, D_{x}\right)$, where $H\left(t, x, \lambda, D_{x}\right)$ is the operator with the symbol $H(t, x, \lambda, \xi)$ and o means an operator product. By Proposition 2.2, if $\rho(t)$ satisfies

$$
0 \leq \rho(t) \leq\left(24^{s} d r\right)^{-\kappa} \quad \text { in } \quad[0, T],
$$

then $A_{\Lambda}(t, x, \xi)$ is in $S_{l^{\prime}}^{1}$, so $H(t, \lambda)$ is in $S_{l^{\prime}}^{N-1}$, where $l^{\prime}$ satisfies (2.8). Since we can take $l$ in (2.8) arbitarly, $H(t, \lambda) \in \cap_{l^{\prime}>0} S_{l^{\prime}}^{N-1}=S^{N-1}$ that is,

$$
\left|H_{(\beta)}^{(\alpha)}(t, x, \lambda, \xi)\right| \leq C\left(|\lambda|+\langle\xi\rangle_{h}\right)^{N-1-|\alpha|},
$$

for $(t, x, \lambda, \xi) \in[0, T] \times \mathbb{R}^{d} \times \mathbb{C} \times \mathbb{R}^{d}, \alpha, \beta \in \mathbb{Z}_{+}^{d}$. 
Denote by $\tilde{S}_{\rho, \delta}^{m}$ a set of symbols $a(t, x, \lambda, \xi)$ which are holomorphic in $\lambda \in$ $\{\lambda \in \mathbb{C}: \operatorname{Im} \lambda \leq 0\}$ and satisfying

$\left|\partial_{\xi}^{\alpha} D_{x}^{\beta} a(t, x, \lambda, \xi)\right| \leq C_{\alpha \beta}\langle\xi\rangle_{h}^{m-\rho|\alpha|+\delta|\beta|}, x, \xi \in \mathbb{R}^{d}, \lambda \in \mathbb{C}, \operatorname{Im} \lambda \leq 0, \alpha, \beta \in \mathbb{Z}_{+}^{d}$.

Next we shall compare $p_{L}(t, x, \lambda, \xi)$ and

$\left.p_{\Lambda}(t, x, \lambda, \xi)=\sigma\left(e^{\Lambda} p\left(t, x, \lambda+i \rho(t)\left\langle D_{x}\right\rangle_{h}^{\kappa}\right), D_{x}\right) e^{-\Lambda}\right)(t, x, \lambda, \xi)$. Since

$$
p(t, x, \lambda, \xi)=\sum_{\pi \in S_{N}} \operatorname{sgn} \pi \cdot a_{1 \pi(1)} a_{2 \pi(2)} \cdots a_{N \pi(N)},
$$

where $a_{i j}=a_{i j}(t, x, \lambda, \xi)$ is the $(i, j)$-component of $(\lambda I-A(t, x, \xi))$ and $S_{N}$ stands for the set of permutations of $\{1,2, \cdots, N\}$, we can write

$$
\begin{aligned}
p\left(t, x, \lambda, D_{x}\right)= & \sum_{\pi \in S_{N}} \operatorname{sgn} \pi \\
& \times a_{1 \pi(1)}\left(x, \lambda, D_{x}\right) \circ a_{2 \pi(2)}\left(x, \lambda, D_{x}\right) \circ \cdots \circ a_{N \pi(N)}\left(x, \lambda, D_{x}\right)+q_{1}
\end{aligned}
$$

and consequently

$$
\begin{aligned}
p_{\Lambda}\left(t, x, \lambda, D_{x}\right)= & \sum_{\pi \in S_{N}} \operatorname{sgn} \pi a_{1 \pi(1), \Lambda}\left(x, D_{x}\right) \circ a_{2 \pi(2), \Lambda}\left(x, D_{x}\right) \circ \cdots \\
& \cdots \circ a_{N \pi(N), \Lambda}\left(x, D_{x}\right)+q_{1 \Lambda},
\end{aligned}
$$

where $a_{j k, \Lambda}(x, D)=e^{\Lambda} a_{j k} e^{-\Lambda}$ and $q_{1}(t, x, \lambda, \xi) \in \tilde{S}_{1,0}^{N-1}$, because

$$
\begin{gathered}
\sigma\left(a_{1 \pi(1), \Lambda}\left(x, D_{x}\right) \circ a_{2 \pi(2), \Lambda}\left(x, D_{x}\right) \circ \cdots \circ a_{N \pi(N), \Lambda}\left(x, D_{x}\right)\right)(x, \xi) \\
=a_{1 \pi(1), \Lambda}(x, \xi) a_{2 \pi(2), \Lambda}(x, \xi) \cdots a_{N \pi(N), \Lambda}(x, \xi)+q_{\pi}(x, \xi)
\end{gathered}
$$

for any $\pi \in S_{N}$, where $q_{\pi}(t, x, \lambda, \xi) \in \tilde{S}_{1,0}^{N-1}$. Hence we have

$$
p_{\Lambda}(t, x, \lambda, \xi)=p_{L}(t, x, \lambda, \xi)+q_{2}(t, x, \lambda, \xi),
$$

where $q_{2} \in \tilde{S}_{1,0}^{N-1}$ that is,

$$
\left|q_{2}^{(\alpha)}(t, x, \lambda, \xi)\right| \leq C_{\alpha \beta}(|\lambda|+\langle\xi\rangle)^{N-1-|\alpha|},
$$

for $(t, x, \lambda, \xi) \in[0, T] \times \mathbb{R}^{d} \times \mathbb{C} \times \mathbb{R}^{d}, \Im \lambda \leq 0$ and $\alpha, \beta \in \mathbb{N}^{d}$. Therefore it follows from (4.5), (4.6) and (4.7) that we have

$$
\sigma(\tilde{P})(t, x, \lambda, \xi)=p_{\Lambda}(t, x, \lambda, \xi) I+Q_{1}(t, x, \lambda, \xi),
$$

where $Q_{1}$ satisfies

$$
\left|Q_{1}^{(\alpha)}(t, x, \lambda, \xi)\right| \leq C_{\alpha \beta}\left(|\lambda|+\langle\xi\rangle_{h}^{\kappa}\right)^{N-1-|\alpha|},
$$

for $(t, x, \lambda, \xi) \in[0, T] \times \mathbb{R}^{d} \times \mathbb{C} \times \mathbb{R}^{d}, \operatorname{Im} \lambda \leq 0$ and $\alpha, \beta \in \mathbb{Z}_{+}^{d}$. 
We shall construct the inverse of $\left(\left(\lambda+i \rho^{\prime}(t)\left\langle D_{x}\right\rangle_{h}^{\kappa}\right) I-A_{\Lambda}(\tau)\right)$ of form $\left(\left(\lambda+i \rho^{\prime}(t)\left\langle D_{x}\right\rangle_{h}^{\kappa}\right) I-A_{\Lambda}(\tau)\right)^{-1}=H\left(\tau, \lambda+i \rho^{\prime}(t)\left\langle D_{x}\right\rangle_{h}^{\kappa}\right) \circ(\tilde{P})^{-1}\left(\tau, \lambda+i \rho^{\prime}(t)\left\langle D_{x}\right\rangle_{h}^{\kappa}\right)$, where $(\tilde{P})^{-1}$ is the inverse operator of $\tilde{P}$. Let us show that $(\tilde{P})^{-1}$ exists. Define a operator $S(t, \lambda)=S\left(t, x, \lambda, D_{x}\right)$ as

$$
S\left(t, x, \lambda, D_{x}\right)=I-\tilde{P}\left(t, x, \lambda, D_{x}\right) \circ M\left(t, x, \lambda, D_{x}\right) .
$$

where $M(t, x, \lambda, \xi)=p\left(t, x, \lambda+i \rho^{\prime}(t)\left\langle D_{x}\right\rangle_{h}^{\kappa}, \xi\right)^{-1}$. Noting that $p(\tau, x, \lambda, \xi)$ is elliptic for $|\lambda| \geq M\langle\xi\rangle_{h}$, we can prove the following proposition by use of Proposition 2.3.

Proposition 4.1. Assume that $1<s<v /(v-1)$ and $q$ satisfies $M_{0}(q)<l$, where $M_{0}(q)$ is given in Proposition 2.1 with $\rho=\kappa, \delta=1-\kappa$. Then, there exists $a$ positive constant $h>0$ such that for any $\tau \in[0, T]$ and $\lambda \in \mathbb{C} \quad(\operatorname{Im} \lambda \leq 0)$.

$$
\left|\partial_{\xi}^{\alpha} D_{x}^{\beta} S(t, x, \lambda, \xi)\right| \leq C_{\alpha \beta}\left(|\lambda|+\langle\xi\rangle_{h}\right)^{\nu(1-\kappa)-1-\kappa|\alpha|+(1-\kappa)|\beta|} .
$$

Proof. If $|\lambda| \geq M\langle\xi\rangle_{h}$, then $p\left(t, x, \lambda+i \rho^{\prime}(t)\langle\xi\rangle_{h}^{\kappa}, \xi\right)$ is elliptic for $|\lambda| \geq M\langle\xi\rangle_{h}(M \gg$ $1)$, so we have

$$
\left|p\left(t, x, \lambda+i \rho^{\prime}(t)\langle\xi\rangle_{h}^{\kappa}, \xi\right)\right| \geq c_{0}\left(|\lambda|+\langle\xi\rangle_{h}\right)^{m},
$$

for $|\lambda| \geq M\langle\xi\rangle_{h}$, which implies (4.11) evidently for $|\lambda| \geq M\langle\xi\rangle_{h}$. From (4.8), the symbol of $\tilde{P}\left(t, x, \lambda, D_{x}\right) \circ M\left(t, x, \lambda, D_{x}\right)$ is

$$
\begin{aligned}
& \sigma(\tilde{P}(t, \lambda) \circ M(t, \lambda))(x, \xi) \\
=I+ & \sum_{0<|\gamma|<n} \frac{1}{\gamma !}\left(p_{\Lambda}(t, x, \lambda, \xi)\right)^{(\gamma)}\left(\frac{1}{p\left(t, x, \lambda+i \rho^{\prime}(t)\langle\xi\rangle_{h}^{\kappa}, \xi\right)}\right)_{(\gamma)} I \\
& +\sum_{0 \leq|\gamma|<n} \frac{1}{\gamma !} Q^{(\gamma)}(t, x, \lambda, \xi)\left(\frac{1}{p\left(t, x, \lambda+i \rho^{\prime}(t)\langle\xi\rangle_{h}^{K}, \xi\right)}\right)_{(\gamma)} I+R_{n}(t, x, \lambda, \xi)
\end{aligned}
$$

where $R_{n}$ is in $\tilde{S}_{\kappa, 1-\kappa}^{(1-\kappa) \nu-\kappa n}$. Moreover, by virtue of Proposition 2.2 and Proposition 2.3

$$
\left(p_{\Lambda}\left(t, x, \lambda+i \rho^{\prime}(t)\langle\xi\rangle_{h}^{\kappa}, \xi\right)\right)^{(\gamma)}\left(\frac{1}{p\left(t, x, \lambda+i \rho^{\prime}(t)\langle\xi\rangle_{h}^{\kappa}, \xi\right)}\right)_{(\gamma)} \in \tilde{S}_{\kappa, 1-\kappa}^{(1-2 \kappa)|\gamma|} \subset \tilde{S}_{\kappa, 1-\kappa}^{-\delta_{1}}
$$

for $1 \leq|\gamma| \leq n$, and

$$
Q_{1}^{(\gamma)}(t, x, \lambda, \xi)\left(\frac{1}{p_{\Lambda}}\right)_{(\gamma)}\left(t, x, \lambda+i \rho^{\prime}(t)\langle\xi\rangle_{h}^{\kappa}, \xi\right) I \in \tilde{S}_{\kappa, 1-\kappa}^{(1-\kappa) \nu-1-\kappa|\gamma|} \subset \tilde{S}_{\kappa, 1-\kappa}^{-\delta_{1}}
$$

for $0 \leq|\gamma| \leq n$, where $\delta_{1}=\min \{2 \kappa-1,1-\nu(1-\kappa)\}=1-\nu(1-\kappa)>0$. Therefore we can see $S(t, x, \lambda, \xi)$ is in $\tilde{S}_{\kappa, 1-\kappa}^{-\delta_{1}}$. This implies (4.11). 
From Proposition 4.1 it follows that if we take the parameter $h>0$ sufficiently large, there exists a $(I-S(\tau, \lambda))^{-1}$ as Neumann series

$$
\begin{aligned}
(I-S(\tau, \lambda))^{-1} & =\sum_{j=0}^{\infty} S(\tau, \lambda)^{j} \\
& =\left\{\tilde{P}\left(\tau, x, \lambda, D_{x}\right) \circ M\left(\tau, x, \lambda, D_{x}\right)\right\}^{-1} .
\end{aligned}
$$

Therefore we obtain the inverse operator of $\left.\left(\lambda+i \rho^{\prime}(\tau)\left\langle D_{x}\right\rangle_{h}^{K}\right) I-A_{\Lambda(\rho(\tau))}(\tau)\right)$ as

$$
\begin{aligned}
& \left(\left(\lambda+i \rho^{\prime}(\tau)\left\langle D_{x}\right\rangle_{h}^{\kappa}\right) I-A_{\Lambda(\rho(\tau))}\left(\tau, x, D_{x}\right)\right)^{-1} \\
= & H\left(\tau, x, \lambda, D_{x}\right) \circ M\left(\tau, x, \lambda, D_{x}\right) \circ \sum_{j=0}^{\infty} S\left(\tau, x, \lambda, D_{x}\right)^{j} \\
= & H(\tau) \circ M(\tau)+H(\tau) \circ M(\tau) \circ \sum_{j=1}^{\infty} S(\tau)^{j} .
\end{aligned}
$$

Proposition 2.2 and Proposition 2.3 yield,

$$
\sigma(H(\tau, \lambda) \circ M(\tau, \lambda))(x, \xi)=H(\tau, \lambda, \xi) M(\tau, \lambda, \xi)+Q_{2}(\tau, x, \lambda, \xi),
$$

where $Q_{2} \in \tilde{S}_{\kappa, 1-\kappa}^{\nu(1-\kappa)-1-\kappa}$. Moreover, noting that $\sigma(H \circ M \circ S) \in \tilde{S}_{\kappa \cdot 1-\kappa}^{2 \nu(1-\kappa)-2}$, we get

$$
\begin{gathered}
\sigma\left(\left(\left(\lambda+i \rho^{\prime}(\tau)\left\langle D_{x}\right\rangle_{h}^{\kappa}\right) I-A_{\Lambda(\rho(\tau))}\left(\tau, x, D_{x}\right)\right)^{-1}\right)(\tau, x, \lambda, \xi) \\
=H(\tau, \lambda, \xi) M(\tau, \lambda, \xi)+Q_{3}(\tau, x, \lambda, \xi)
\end{gathered}
$$

where $Q_{3} \in \tilde{S}_{\kappa, 1-\kappa}^{-\delta}$, where $\delta=\min \{1+\kappa-v(1-\kappa), 2(1-v(1-\kappa))\}=$ $2(1-v(1-\kappa))>0$.

\section{The equation with time-independent coefficients}

In this section, we shall solve the following equation: for fixed $\tau \in[0, T)$,

$$
\left\{\begin{array}{l}
P_{\Lambda(\rho(\tau))}\left(\tau, x, D_{t, \Lambda(\rho(\tau))}, D_{x}\right) v(t, x),=g(t, x), \quad T \geq t>\tau, x \in \mathbb{R}^{d} \\
v(\tau, x)=v_{0}(x) .
\end{array}\right.
$$

We define

$$
\begin{aligned}
V_{0}(t, \tau) & =V_{0}\left(t, \tau, x, D_{x}\right) \\
& =\frac{1}{2 \pi i} \int_{\operatorname{Im} \lambda=0} e^{i \lambda(t-\tau)}\left(\left(\lambda+i \rho^{\prime}(\tau)\left\langle D_{x}\right\rangle_{h}^{\kappa}\right) I-A_{\Lambda(\rho(\tau))}\left(\tau, x, D_{x}\right)\right)^{-1} d \lambda
\end{aligned}
$$

for $0 \leq \tau \leq t \leq T$. 
Proposition 5.1. Let $1<s<1+\frac{\mu}{v}, \mu \leq 1, \kappa=1 / s$, and $\sigma=(v-1)(1-\kappa)$. Then there is $\kappa^{\prime}>0$ such that $\kappa<\kappa^{\prime}<1,1-\kappa^{\prime}<\kappa^{\prime}$ and $V_{0}(t, \tau)$ is a pseudo differential operator of which symbol is in $S_{\kappa^{\prime}, 1-\kappa^{\prime}}^{\sigma}$. Moreover there is a constant $C>0$ such that

$$
\left\|V_{0}(t, \tau) u\right\|_{H_{\Lambda(\rho)}^{q}} \leq C\|u\|_{H_{\Lambda(\rho)}^{q+\sigma}}, \quad T \geq t>\tau,
$$

for $u \in H_{\Lambda(\rho)}^{q}$.

Proof. It is sufficient to prove that there is $\kappa^{\prime}>0$ such that $1>\kappa^{\prime}>1-\kappa^{\prime}$ and

$$
\left|\partial_{\xi} D_{x}^{\alpha} V_{0}(t, \tau, x, \xi)\right| \leq C_{\alpha \beta}\langle\xi\rangle_{h}^{\sigma-\kappa^{\prime}|\alpha|+\left(1-\kappa^{\prime}\right)|\beta|}, \quad T \geq t \geq \tau, x, \xi \in \mathbb{R}^{n} .
$$

for $\alpha, \beta \in \mathbb{N}^{d}$. Since it is known from Proposition 3.3 in [6] that $V_{0}(t, \tau)(x, \xi)$ is in $S_{\kappa, 1-\kappa}^{v(1-\kappa)}$, it suffices to

$$
\left|V_{0}(t, \tau)(x, \xi)\right| \leq C\langle\xi\rangle_{h}^{\sigma}, \quad T \geq t \geq \tau, x, \xi \in \mathbb{R}^{n} .
$$

In fact, we can choose $\kappa^{\prime}$ such that $\kappa>\kappa^{\prime}$ and $\kappa^{\prime}>1-\kappa^{\prime}$ because of $\kappa>1-\kappa$. $V_{0}(t, \tau)(x, \xi)$ is in $S_{\kappa, 1-\kappa}^{\nu(1-\kappa)}$ implies

$$
\left|\partial_{\xi} D_{x}^{\alpha} V_{0}(t, \tau, x, \xi)\right| \leq C_{\alpha \beta}\langle\xi\rangle_{h}^{\sigma-\kappa^{\prime}|\alpha|+\left(1-\kappa^{\prime}\right)|\beta|}, \quad T \geq t \geq \tau, x, \xi \in \mathbb{R}^{n} .
$$

for $|\alpha+\beta| \geq \frac{1-\kappa}{\kappa-\kappa^{\prime}}$. Using the interpolation theorem we can get (5.4) from (5.5) and (5.6). Now we shall show (5.5). (5.2) yields

$$
\begin{aligned}
& V_{0}(t, \tau)(x, \xi)=\frac{1}{2 \pi i} \int_{\operatorname{Im} \lambda=0} e^{i \lambda(t-\tau)}\left(\left(\lambda+i \rho^{\prime}(\tau)\left\langle D_{x}\right\rangle_{h}^{\kappa}\right) I-A_{\Lambda}(\rho(\tau))^{-1}(\tau, x, \xi)\right) d \lambda \\
& =\frac{1}{2 \pi i} \lim _{R \rightarrow \infty} \int_{\operatorname{Im} \lambda=0,|\operatorname{Re} \lambda| \leq R} e^{i \lambda(t-\tau)}\left(\left(\lambda+i \rho^{\prime}(\tau)\left\langle D_{x}\right\rangle_{h}^{\kappa}\right) I-A_{\Lambda}(\rho(\tau))^{-1}(\tau, x, \xi)\right) d \lambda .
\end{aligned}
$$

Besides,

$$
\begin{aligned}
\left.\int_{\operatorname{Im} \lambda=0,|\operatorname{Re} \lambda| \leq R} e^{i \lambda(t-\tau)}\left(\left(\lambda+i \rho^{\prime}(\tau)\left\langle D_{x}\right\rangle_{h}^{\kappa}\right) I-A_{\Lambda(\rho(\tau)}\right)^{-1}(\tau, x, \xi)\right) d \lambda \\
\left.=\int_{C_{R}} e^{i \lambda(t-\tau)}\left(\left(\lambda+i \rho^{\prime}(\tau)\left\langle D_{x}\right\rangle_{h}^{\kappa}\right) I-A_{\Lambda(\rho(\tau))}\right)^{-1}(\tau, x, \xi)\right) d \lambda \\
\left.\quad-\int_{\gamma_{R}} e^{i \lambda(t-\tau)}\left(\left(\lambda+i \rho^{\prime}(\tau)\left\langle D_{x}\right\rangle_{h}^{\kappa}\right) I-A_{\Lambda(\rho(\tau)))}\right)^{-1}(\tau, x, \xi)\right) d \lambda
\end{aligned}
$$

where $C_{R}=\{\lambda \in \mathbb{C} ; \operatorname{Im} \lambda=0,|\operatorname{Re} \lambda| \leq R\} \cup \gamma_{R}, \gamma_{R}=\{|\lambda|=R, \operatorname{Im} \lambda \leq 0\}$. Noting

$$
\left.\lim _{R \rightarrow \infty} \int_{\gamma_{R}} e^{i \lambda(t-\tau)}\left(\left(\lambda+i \rho^{\prime}(\tau)\left\langle D_{x}\right\rangle_{h}^{\kappa}\right) I-A_{\Lambda(\rho(\tau))}\right)^{-1}(\tau, x, \xi)\right) d \lambda=0
$$


we get

$$
\begin{aligned}
V_{0}(t, \tau)(x, \xi)= & \frac{1}{2 \pi i} \lim _{R \rightarrow \infty} \int_{\operatorname{Im} \lambda=0,|\operatorname{Re} \lambda| \leq R} e^{i \lambda(t-\tau)}\{H(\tau, x, \lambda, \xi) M(\tau, x, \lambda, \xi) \\
=: & \left.+Q_{3}(\tau, x, \lambda, \xi)\right\} d \lambda
\end{aligned}
$$

where

$$
I_{1 R}(x, \xi)=\frac{1}{2 \pi i} \int_{C_{R}} e^{i \lambda(t-\tau)} \frac{H(\tau, x, \lambda, \xi)}{p\left(\tau, x, \lambda+i \rho^{\prime}(\tau)\langle\xi\rangle_{h}^{\kappa}, \xi\right)} d \lambda
$$

and

$$
I_{2 R}(x, \xi)=\frac{1}{2 \pi i} \int_{C_{R}} e^{i \lambda(t-\tau)} Q_{3}(\tau, x, \lambda, \xi) d \lambda
$$

Noting that the roots of $p\left(t, x, \lambda+i \rho^{\prime}(\tau)\langle\xi\rangle_{h}^{\kappa}, \xi\right)=0$ are contained in the domaim $C_{R}$ for large $R$, we can prove similarly as Proposition 2.1 in [11] that $I_{1 R}(x, \xi)$ satisfies (5.5). Next we prove that $I_{2 R}(x, \xi)$ satisfies (5.5). Since $Q_{3}$ satisfies from (4.14)

$$
\left|Q_{3}(\tau, x, \lambda, \xi)\right| \leq C\left(|\lambda|+\langle\xi\rangle_{h}\right)^{2(v(1-\kappa)-1)}
$$

we can estimate

$$
\begin{aligned}
\left|I_{2 R}(x, \xi)\right| & =\left|\frac{1}{2 \pi i} \int_{C_{R}} e^{i \lambda(t-\tau)} Q_{3}(\tau, x, \lambda, \xi) d \lambda\right| \\
& \leq C \int_{|\operatorname{Im} \lambda| \leq R}\left(|\lambda|+\langle\xi\rangle_{h}\right)^{2(\nu(1-\kappa)-1)} d \operatorname{Im} \lambda \\
& \leq C\langle\xi\rangle_{h}^{2(\nu(1-\kappa)-1)+1} \leq C\langle\xi\rangle_{h}^{(\nu-1)(1-\kappa)} .
\end{aligned}
$$

Here we used the inequality $2(v(1-\kappa)-1)+1 \leq(v-1)(1-\kappa)$ which is implied by the assumption $\kappa>\frac{v}{v+\mu} \geq \frac{v}{v+1}$. Thus we compleled the proof of Proposition 5.1.

It is easily seen that $V_{0}(t, \tau)$ satisfies

$$
\left\{\begin{array}{l}
D_{t} V_{0}(t, \tau)=\left\{-i \rho^{\prime}(\tau)\left\langle D_{x}\right\rangle_{h}^{\kappa}+A_{\Lambda(\rho(\tau))}\left(\tau, x, D_{x}\right)\right\} V_{0}(t, \tau) \quad(\tau<t \leq T) \\
V_{0}(\tau, \tau)=\tilde{I}
\end{array}\right.
$$

where $\tilde{I}$ means the identity operator. Thus we have obtained a solution $v(t)$ of (5.1) as follows:

$$
v(t)=V_{0}(t, 0) v_{0}+\int_{0}^{t} V_{0}(t, r) g(r) d r
$$




\section{Construction of the semi-group}

We shall construct a semi-group $V(t, \tau)=V\left(t, \tau, x, D_{x}\right)$ for the generator $\rho^{\prime}(t)\left\langle D_{x}\right\rangle_{h}^{\kappa}-i A_{\Lambda(\rho(t))}\left(t, x, D_{x}\right)+B(t, x)$;

$$
\left\{\begin{array}{l}
D_{t} V(t, x ; \tau)=\left\{-i \rho^{\prime}(t)\left\langle D_{x}\right\rangle_{h}^{\kappa}+A_{\Lambda(\rho(t))}\left(t, x, D_{x}\right)+i B(t, x)\right\} V(t, x ; \tau), \\
\quad t>\tau \geq 0 \\
V(\tau, \tau)=I
\end{array}\right.
$$

We note that $V(t, \tau)$ has a semi group property, that is, $V(t, \tau)=V(t, r) V(r, \tau)$ for $T \geq t \geq r \geq \tau \geq 0$. In order to construct $V(t, \tau)$, we shall use TanabeSobolevski's method and Banach scale method (cf. $[6,10])$.

We shall seek the pseudo differential operator $V(t, \tau)=V\left(t, \tau, x, D_{x}\right)$ which satisfies the following equation:

$$
V\left(t, \tau, x, D_{x}\right)=V_{0}\left(t, \tau, x, D_{x}\right)+\int_{\tau}^{t} V_{0}\left(t, r, x, D_{x}\right) \Phi\left(r, \tau, x, D_{x}\right) d r .
$$

If $V(t, \tau)$ satisfies $(6.1), \Phi(t, x, D ; \tau)$ must satisfy the following equation:

$$
\Phi\left(t, \tau, x, D_{x}\right)=R\left(t, \tau, x, D_{x}\right)+\int_{\tau}^{t} R\left(t, r, x, D_{x}\right) \Phi\left(r, \tau, x, D_{x}\right) d r
$$

where

$$
\begin{aligned}
R\left(t, \tau, x, D_{x}\right)= & \left\{-i \rho^{\prime}(t)\left\langle D_{x}\right\rangle_{h}^{\kappa}+A_{\Lambda(\rho(t))}\left(t, x, D_{x}\right)+i \rho^{\prime}(\tau)\left\langle D_{x}\right\rangle_{h}^{\kappa}\right. \\
& \left.-A_{\Lambda(\rho(\tau))}\left(\tau, x, D_{x}\right)+i B(t, x)\right\} \circ V_{0}\left(t, \tau, x, D_{x}\right) .
\end{aligned}
$$

Conversely, if $\Phi\left(t, \tau, x, D_{x}\right)$ satisfies (6.3), then $V\left(t, \tau, x, D_{x}\right)$ satisfies (6.2).

We shall construct a solution $\Phi\left(t, \tau, x, D_{x}\right)$ of the equation (6.3) as follows:

$$
\Phi\left(t, \tau, x, D_{x}\right)=\sum_{j=0}^{\infty} \Phi_{j}\left(t, \tau, x, D_{x}\right)
$$

where

$$
\left\{\begin{array}{l}
\Phi_{0}\left(t, \tau, x, D_{x}\right)=R\left(t, \tau, x, D_{x}\right), \\
\Phi_{j}\left(t, \tau, x, D_{x}\right)=\int_{\tau}^{t} R\left(t, r, x, D_{x}\right) \circ \Phi_{j-1}\left(r, \tau, x, D_{x}\right) d r \quad(j \geq 1) .
\end{array}\right.
$$

For simplicity of notation denote $H_{\Lambda(\rho)}^{q}$ by $H_{\rho}^{q}$. We recall

$$
\|u\|_{H_{\rho}^{q}}=\left\|\left\langle D_{x}\right\rangle_{h}^{q} e^{\rho\left\langle D_{x}\right\rangle_{h}^{\kappa}} u\right\|_{L^{2}} \quad \kappa=\frac{1}{s} .
$$


We know that for $l \geq 0$ and for $\rho_{0} \geq \rho^{\prime}>\rho \geq 0$

$$
\left\|\left\langle D_{x}\right\rangle_{h}^{l} u\right\|_{H_{\rho}^{q}} \leq C\left(\rho^{\prime}-\rho\right)^{-s l}\|u\|_{H_{\rho^{\prime}}^{q}}
$$

Let denote $\sigma=(\nu-1)(1-\kappa)$ and take $\delta \geq 0$ such that $1>s(\sigma+\delta), 1+\mu>$ $s(1+\sigma+\delta)$. It is possible, because of the assumption $s \sigma<1$ and $1+\mu>s(1+\sigma)$. Denote $s_{1}=s(\sigma+\delta+1), s_{0}=s(\sigma+\delta)$ and $\epsilon=\min \left\{1-s_{0}, 1+\mu-s_{1}\right\}=$ $1+\mu-s_{1}>0$.

Proposition 6.1. There is $C_{R}>0$ such that for $T \geq t \geq \tau \geq 0$ and for $\rho_{0} \geq \rho^{\prime}>$ $\rho \geq 0$

$$
\|R(t, \tau) u\|_{H_{\rho}^{q+\delta}} \leq C_{R}\left\{|t-\tau|^{\mu}\left(\rho^{\prime}-\rho\right)^{-s_{1}}+\left(\rho^{\prime}-\rho\right)^{-s_{0}}\right\}\|u\|_{H_{\rho^{\prime}}^{q}}
$$

Proof. It follows from (6.4), (5.3) and (6.7) that

$$
\begin{aligned}
& \|R(t, \tau) u\|_{H_{\rho}^{q+\delta}} \leq C_{R}\left(|t-\tau|^{\mu}||\left\langle D_{x}\right\rangle_{h}^{1+\delta+\sigma} u\left\|_{H_{\rho}^{q}}+\right\|\left\langle D_{x}\right\rangle_{h}^{\delta+\sigma} u \|_{H_{\rho}^{q}}\right) \\
& \leq C_{R}\left\{|t-\tau|^{\mu}\left(\rho^{\prime}-\rho\right)^{-s_{1}}+\left(\rho^{\prime}-\rho\right)^{-s_{0}}\right\}\|u\|_{H_{\rho^{\prime}}^{q}}
\end{aligned}
$$

for $\rho_{0} \geq \rho^{\prime}>\rho \geq 0$, which proves proposition.

Now we shall prove that (6.5) is convergent.

Proposition 6.2. There are $C_{1}>0, C_{2}>0$ such that for $u \in H_{\rho^{\prime}}^{q}, T \geq t \geq \tau \geq 0$, and for $\rho_{0} \geq \rho^{\prime}>\rho \geq 0$

$$
\begin{aligned}
& \left\|\Phi_{j}(t, \tau) u\right\|_{H_{\rho}^{q+\delta}} \leq C_{j}(t-\tau)^{j}\left\{\frac{(t-\tau)^{\mu(j+1)}}{\left(\rho^{\prime}-\rho\right)^{s_{1}(j+1)}}+\frac{1}{\left(\rho^{\prime}-\rho\right)^{s_{0}(j+1)}}\right\}\|u\|_{H_{\rho^{\prime}}^{q+\delta}}, \\
& j=0,1, \cdots \text {, }
\end{aligned}
$$

where $s_{1}=v(s-1)+s$ and $s_{0}=(v-1)(s-1)$ and

$$
C_{j}=C_{1} C_{2}^{j}(j !)^{-\epsilon}, \quad \epsilon=\min \left\{1-s_{0}, 1+\mu-s_{1}\right\}=1+\mu-s_{1}>0 .
$$

Proof. We shall prove this augument (6.9) by induction. Proposition 6.1 assures (6.9) for $j=0$. Assume that (6.9) holds for $j-1$. Then from (6.6) and (6.8)

$$
\begin{aligned}
\left\|\Phi_{j}\left(t, \tau, x, D_{x}\right) u\right\|_{H_{\rho}^{q+\delta}} \leq \int_{\tau}^{t}\left\|R\left(t, r, x, D_{x}\right) \circ \Phi_{j-1}\left(r, \tau, x, D_{x}\right) u\right\|_{H_{\rho}^{q+\delta}} d r \\
\leq C_{R} \int_{\tau}^{t}\left\{\frac{|t-r|^{\mu}}{\left(\rho^{\prime \prime}-\rho\right)^{s_{1}}}+\frac{1}{\left(\rho^{\prime \prime}-\rho\right)^{s_{0}}}\right\} \\
\quad \times C_{j-1}(r-\tau)^{j-1}\left\{\frac{(r-\tau)^{\mu j}}{\left(\rho^{\prime}-\rho^{\prime \prime}\right)^{s_{1} j}}+\frac{1}{\left(\rho^{\prime}-\rho^{\prime \prime}\right)^{s_{0} j}}\right\} d r\|u\|_{H_{\rho^{\prime}}^{q}}
\end{aligned}
$$


for $\rho_{0} \geq \rho^{\prime}>\rho^{\prime \prime}>\rho \geq 0$. Here we choose $\rho^{\prime \prime}=\frac{\rho^{\prime}+j \rho}{j+1}$. Taking account of the following equalities and inequalities

$$
\frac{1}{\left(\rho^{\prime \prime}-\rho\right)^{p}\left(\rho^{\prime \prime}-\rho\right)^{q}}=\frac{(a+b)^{p+q}}{a^{p} b^{q}}\left(\rho^{\prime}-\rho\right)^{-p-q},
$$

where $\rho^{\prime \prime}=\frac{a \rho+b \rho^{\prime}}{a+b}, 0<\rho<\rho^{\prime}$ and $a>0, b>0, p>0, q>0$,

$$
\int_{\tau}^{t}(t-r)^{a}(r-\tau)^{b} d r=(t-\tau)^{a+b+1} \frac{\Gamma(a+1) \Gamma(b+1)}{\Gamma(a+b+2)},
$$

where $a>-1, b>-1$,

$$
(A+B)\left(A^{k}+B^{k}\right) \leq 4\left(A^{k+1}+B^{k+1}\right),
$$

where $A, B>0$ and $k$ a positive integer and

$$
\left(1+\frac{1}{j}\right)^{j} \leq e, \quad j=1,2, \cdots
$$

we can get from (6.11)

$$
4 e^{s_{1}} C_{j-1} C_{R} \max \left\{\frac{(j+1)^{s_{1}} \Gamma(j(\mu+1))}{\Gamma((j+1)(\mu+1)}, \frac{(j+1)^{s_{1}} \Gamma(j)}{\Gamma(j+\mu+1)},(j+1)^{s_{0}-1}\right\} \leq C_{j} .
$$

By virture of the assumption $s<1+\frac{\mu}{v}$ we can take $\epsilon=\min \left\{1+\mu-s_{1}, 1-s_{0}\right\}=$ $1+\mu-s_{1}>0$ such that

$$
\max \left\{\frac{(j+1)^{s_{1}} \Gamma(j(\mu+1))}{\Gamma((j+1)(\mu+1))}, \frac{(j+1)^{s_{1}} \Gamma(j)}{\Gamma(j+\mu+1)},(j+1)^{s_{0}-1}\right\} \leq C j^{-\epsilon} .
$$

Therefore we can find $C_{1}, C_{2}$ such that $C_{j}$ given by (6.10) satisfies (6.12).

From Proposition 6.2, we can observe that $\Phi\left(t, \tau, x, D_{x}\right)$ is well-defined by (6.5), so $V\left(t, \tau, x, D_{x}\right)$ can be also defined by (6.2). Precisely,

Proposition 6.3. Let $q \in \mathbb{R}, u \in H_{\rho^{\prime}}^{q}, T \geq t \geq \tau \geq 0$ and $\rho_{0} \geq \rho^{\prime}>\rho \geq 0$. Denote $s_{1}=s(\sigma+\delta+1), s_{0}=s(\sigma+\delta)$ and $\epsilon=\min \left\{1-s_{0}, 1+\mu-s_{1}\right\}=1+\mu-s_{1}$.

(i) There exists a constant $C_{2}>0$ such that

$$
\begin{aligned}
& \|\Phi(t, x ; \tau) u\|_{H_{\rho}^{q+\delta}} \\
& \leq C_{2}\left(\frac{(t-\tau)^{\mu}}{\left(\rho^{\prime}-\rho\right)^{s_{1}}}+\frac{1}{\left(\rho^{\prime}-\rho\right)^{s_{0}}}\right) \exp \left\{\left[C_{2} \frac{(t-\tau)^{\mu+1}}{\left(\rho^{\prime}-\rho\right)^{s_{1}}}+\frac{t-\tau}{\left(\rho^{\prime}-\rho\right)^{s_{0}}}\right]^{1 / \epsilon}\right\}\|u\|_{H_{\rho^{\prime}}^{q}}
\end{aligned}
$$


(ii) There exists a constant $C_{3}>0$ such that

$$
\begin{aligned}
\|V(t, x ; \tau) u\|_{H_{\rho}^{q+\delta}} & \leq C_{3}\left\{\left(\rho^{\prime}-\rho\right)^{-s_{0}}+\left(\frac{(t-\tau)^{1+\mu}}{\left(\rho^{\prime}-v \rho\right)^{s_{1}+s \sigma}}+\frac{t-\tau}{\left(\rho^{\prime}-\rho\right)^{s_{0}+s \sigma}}\right)\right. \\
& \left.\times \exp C_{3}\left(\frac{(t-\tau)^{1+\mu}}{\left(\rho^{\prime}-\rho\right)^{s_{1}}}+\frac{(t-\tau)}{\left(\rho^{\prime}-\rho\right)^{s_{0}}}\right)^{1 / \epsilon}\right\}\|u\|_{H_{\rho^{\prime}}^{q}}
\end{aligned}
$$

Proof. (i): We note that for a positive integer $j$ and $x>0$

$$
\sup _{x \geq 0} x^{j} e^{-x}=\frac{x^{j}}{\sum_{n=0}^{\infty} x^{n} n !^{-1}}<j !
$$

implies that $\frac{1}{j ! \epsilon} \leq \frac{e^{\epsilon}}{x^{\epsilon j}}$ for $x>0, \epsilon>0$ and if we take $x=(2 A)^{\frac{1}{\epsilon}}$

$$
\sum_{j=0}^{\infty} \frac{A^{j}}{j ! \epsilon} \leq 2 e^{\epsilon(2 A)^{\frac{1}{\epsilon}}}
$$

for $A>0, \epsilon>0$. If we take $A$ like

$$
A=C_{2}\left\{\frac{(t-\tau)^{1+\mu}}{\left(\rho^{\prime}-\rho\right)^{s_{1}}}+\frac{(t-\tau)}{\left(\rho^{\prime}-\rho\right)^{s_{0}}}\right\}
$$

we can see that (6.13) holds.

(ii): $\quad$ (6.14) is a direct result of (5.3), (6.2) and of (6.13).

Put

$$
v(t)=V(t, 0) v_{0}+\int_{0}^{t} V(t, r) g(r) d r
$$

then, it is obvious that $v(t)$ is a solution of the equation (3.1), moreover, it follows from (6.14) that if we take $\rho^{\prime}=\rho_{0}, \rho=0$, there is a constant $C>0$ such that for $T \geq t>0$

$$
\|v(t, \cdot)\|_{H^{q+\delta}} \leq C\left(\left\|v_{0}\right\|_{H_{\rho_{0}}^{q}}+\int_{0}^{t}\|g(r, \cdot)\|_{H_{\rho_{0}}^{q}} d r\right) .
$$

Since $u(t, x)=e^{-\Lambda(T-t)} v(t, x)$ satisfies (1.1) and (6.15) implies (1.2), we have completed the proof of Theorem 1.7. 


\section{References}

[1] M. D. BRonshtein, Cauchy problem for hyperbolic operators with variable multiple characteristics (Russian), Trudy Moscow Math. 41 (1980), 83-99.

[2] F. Colombini, E. JANnelli and S. SPAgnolo, Wellposedness in the Gevrey classes of the Cauchy problem for a non strictly hyperbolic equation with coefficients depending on time, Ann. Scuola Norm. Sup. Pisa Cl. Sci. 10 (1983), 291-312.

[3] P. D'AnCona, T. KinoshitA and S. SPAGNOLO, Weakly hyperbolic systems with Hölder continuous coefficients, J. Differential Equations 203 (2004), 64-81.

[4] K. KAJITANI, Local solution of Cauchy problem for nonlinear hyperbolic systems in Gevrey classes, Hokkaido Math. J. 23-3 (1983), 599-616.

[5] P. D'Ancona, T. Kinoshita and S. SPAGnolo, Cauchy problem for nonstrictly hyperbolic systems in Gevrey classes, J. Math. Kyoto. Univ. 12 (1983), 434-460.

[6] P. D'Ancona, T. Kinoshita and S. Spagnolo, The Cauchy Problem for Nonlinear Hyperbolic Systems, Bull. Sci. Math. 110 (1986), 3-48.

[7] P. D'Ancona, T. Kinoshita and S. Spagnolo, Wellposedness in Gevrey class of the Cauchy problem for hyperbolic operators, Bull. Sc. Math. 111 (1987), 415-438.

[8] T. Nishitani, Sur les équations hyperboliques à coefficients hölderients en t et de classes de Gevrey en x, Bull. Sci. Math. 107 (1983), 113-138.

[9] Y. OHYA and S. TARAma, Le Problème de Cauchy à caractéristiques multiples dans la classe de Gevrey - coefficients hölderiens en $t$-, In: "Hyperbolic Equations and Related Topics", Proc. Taniguchi Internat. Symp. (1984), 273-302.

[10] H. TANABE, "Equations of Evolution", translated from the Japanese by N. Mugibayashi and H. Haneda. Monographs and Studies in Mathematics, Vol. 6, Pitman (Advanced Publishing Program), Boston, Mass.-London, 1979.

[11] Y. YUZAWA, The Cauchy problem for hyperbolic systems with Hölder continuous coefficients with respect to time, J. Differential Equations 219 (2005), 363-374.

Institute of Mathematics

Universitiy of Tsukuba

1-1-1 Tennoudai, Tsukuba

Ibaraki 305-8571, Japan

kajitani@math.tsukuba.ac.jp

Nippon Institute of Technology

4-1 Gakuen-dai Miyashiro-cho Minami-Saitama

Saitama, Japan 